\title{
A vascular cause of neck pain
}

\author{
Brian Reed Curtis ${ }^{1} \cdot$ Evan Fitchett $^{2}$
}

Received: 28 October 2016/Accepted: 10 December 2016/Published online: 24 December 2016

(C) SIMI 2016

\section{History and presentation}

A 55-year-old woman without any significant past medical history presented to her primary care physician with neck pain and tingling in her left arm. There was no history of trauma. On physical examination, she had left-sided neck pain. The upper extremity strength and reflexes were normal, and the cranial nerves were intact. There were no visual field defects and no history of vision loss. The pupils were equal in size, and were reactive at the time of the visit with her physician. Her pain was partially relieved with a short course of steroids, but she continued to have numbness and tingling in her left arm. One week prior to MRI, she began to have headaches, and 1 day prior to the MRI, she noticed her pupils were uneven; her left pupil was smaller than the right. She also began to have visual scintillations.

\section{Imaging}

A cervical spine MRI shows no significant degenerative changes or disc herniations to explain the patient's pain. However, there is an eccentric, crescent-shaped hyperintense signal encasing the anterior aspect of the left internal carotid artery on T1- and T2-weighted MRI images, which

Brian Reed Curtis

brian.curtis@jefferson.edu

1 Department of Radiology, Thomas Jefferson University Hospital, 132 South 10th Street, Philadelphia, PA 19107, USA

2 Sidney Kimmel Medical College, Thomas Jefferson University, Philadelphia, USA is much more conspicuous on T1 imaging with fat suppression (Fig. 1). This finding is characteristic of a subintimal hematoma related to left internal carotid artery dissection. The proximal carotid arteries demonstrate a normal MRI appearance for comparison (Fig. 2).

\section{Clinical course}

The patient was brought to the hospital where she was evaluated by neurology and neurosurgery. She remained stable throughout treatment without signs of infarction or other complication from her internal carotid artery dissection. Although the spontaneity of our patient's carotid dissection suggests a familial arterial disease, none has been confirmed.

\section{Discussion}

There are many causes for neck pain, most of which are musculoskeletal in nature, including muscular or soft tissue etiologies, fractures, degenerative disc disease, and disc herniations. Although relatively rare with an incidence of approximately 5 in 100,000, dissection of the carotid or vertebral arteries is an important cause of neck pain [1]. Craniocervical arterial dissection occurs more often in patients with familial arterial disease such as fibromuscular dysplasia. Often, there is an inciting event, which can range from significant trauma to chiropractic maneuvers, or even minor events such as bending over. However, arterial dissection can rarely occur spontaneously.

Carotid artery dissection most often involves the extracranial internal carotid artery. It can present with a 


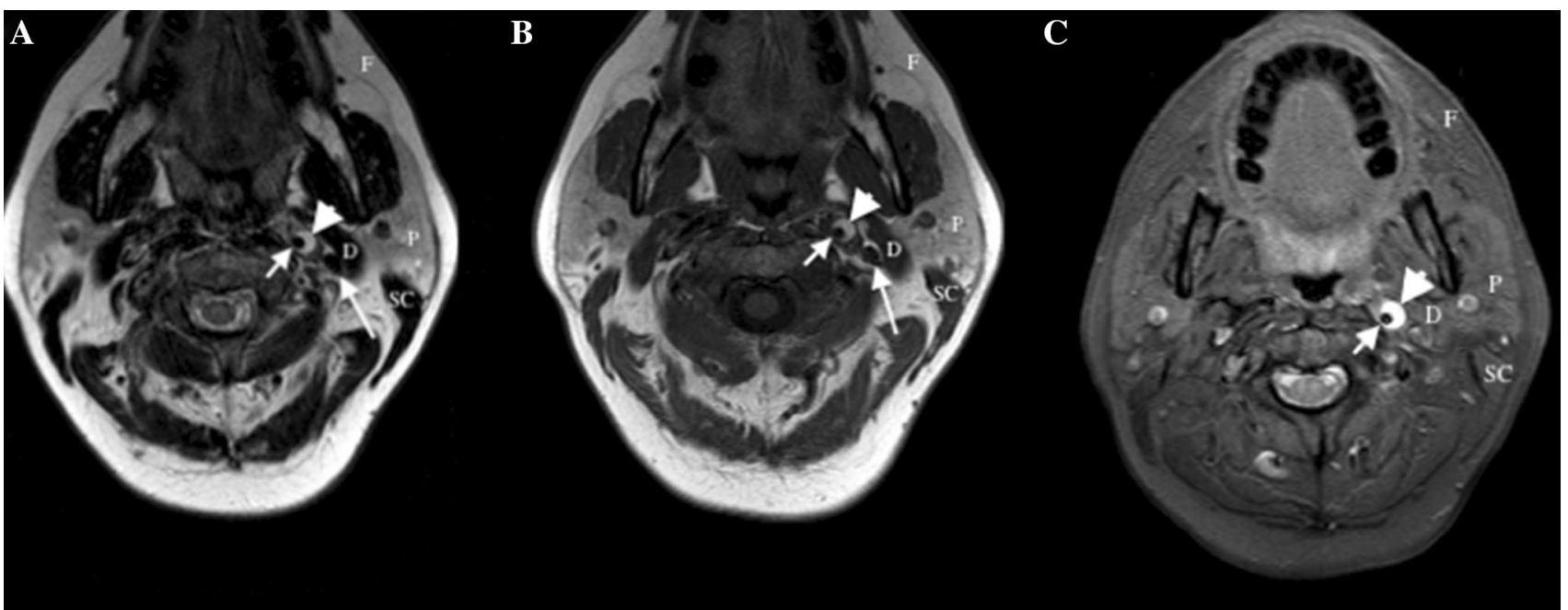

Fig. 1 Axial T2-weighted (a), T1-weighted (b) and T1-weighted fatsuppressed (c) MR images at the level of the parotid glands $(P)$ demonstrate left internal carotid artery dissection. a-c Crescentshaped bright signal caused by sub-intimal blood products (arrowhead) draped over the black left internal carotid artery flow void (short arrow). Internal jugular vein flow void is noted (long arrow) just medial to the posterior belly of the digastric muscle $(D)$. c Darkening of fat $(F)$, which makes the bright crescent-shaped left internal carotid artery sub-intimal hematoma (arrowhead) conspicuous. This is characteristic of arterial dissection. ( $S C$ - sternocleidomastoid muscle)

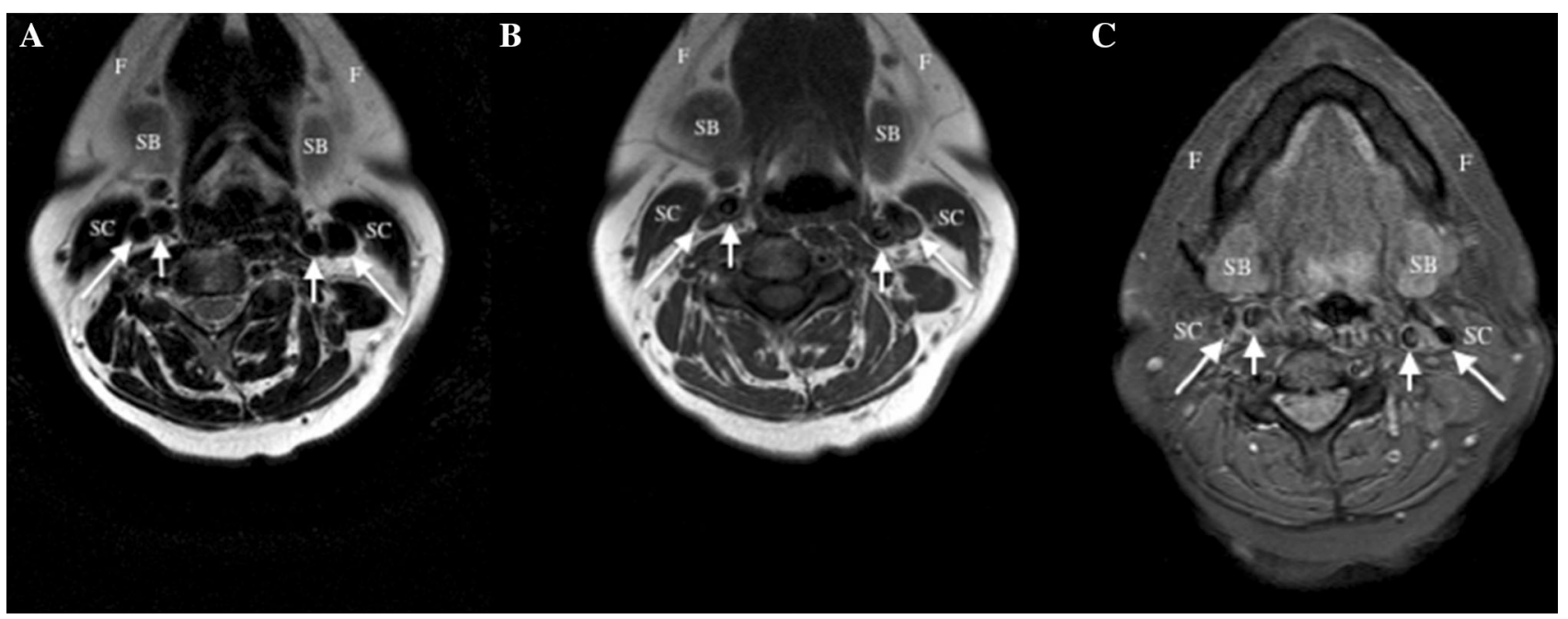

Fig. 2 Axial T2-weighted (a), T1-weighted (b) and T1-weighted fatsuppressed (c) MR images of the same patient in Fig. 1 at the level of the submandibular glands $(S B)$ show normal flow voids in the more proximal carotid arteries. a-c Normal black flow voids in the carotid arteries (short arrows) without surrounding crescent-shaped bright

partial Horner's syndrome with miosis and ptosis. Miosis and oculosympathetic paresis are results of altered sympathetic pathways in the neck that normally contribute to pupil dilatation and partial elevation of the eyelid. In fact, one study reports that $44 \%$ of patients with internal carotid artery dissection presented with a painful Horner syndrome. Furthermore, 28\% have transient monocular vision abnormalities with scintillations, which our patient also eventually experienced, and this is attributed to choroidal hypoperfusion [2]. These are important signs and signal. The adjacent internal jugular vein flow voids (long arrows) are just medial to the sternocleidomastoid muscles $(S C)$. Note the darkening of fat $(F)$ in $\mathbf{c}$ and the lack of bright signal surrounding the normal vasculature

symptoms to evaluate for while performing a history and physical examination on patients with neck pain.

An MRI is commonly ordered to evaluate neck pain, especially when there are neurologic symptoms, since its contrast resolution allows outstanding soft tissue characterization of disc herniations, nerve impingement and other common musculoskeletal disorders. Clinicians may not suspect arterial dissection and dedicated vascular imaging may not be ordered; however, arterial dissection can be diagnosed on non-contrast cervical spine MRI imaging 
with diligent observation. Therefore, it is important for all physicians interpreting imaging to specifically evaluate the neck vasculature.

Patent blood vessels with fast flowing blood create "flow voids" on MRI that appear a black signal along the vessel course [3]. This artifact is related to protons in the blood moving out of the region imaged in between the radiofrequency excitation pulse and the time that the excited protons relax, and release the radiofrequency that is measured to produce an image. This is a helpful artifact; if it is present, then the vessel can be considered patent, especially on T2-weighted images. Loss of a flow void can represent vascular occlusion or slow blood flow. Our patient maintains a flow void in her left internal carotid artery indicating luminal patency, but there is an abnormal signal surrounding its anterior aspect on T1- and T2weighted images. Hyperintense signal on T1-weighted images is caused by few materials found in the human body including blood products, fat, melanin, proteinaceous material, calcification and other mineralization. In vascular dissection, a tear in the tunica intima allows blood to dissect into the sub-intimal space and into the tunica media. Extravascular blood products evolve from hemoglobin to methemoglobin, a change that is observed by signal intensity change on MRI. Subacute hemorrhage with methemoglobin appears bright on $\mathrm{T} 1$ images; this is seen around 7 days after the formation of the initial hematoma, and remains up to 2 months [4]. The crescent-shaped T1 hyperintensity surrounding the anterior aspect of the left internal carotid artery is diagnostic of a sub-intimal hematoma related to a tear in the tunica intima that remains contained by the tunica adventitia. This finding is confirmed and more conspicuous on T1 fat-saturated images, since the abundant normal $\mathrm{T} 1$ hyperintense fat is darkened and T1 hyperintense blood products remain bright. This can easily be overlooked on MRI, especially without fatsuppressed T1 images (which are not typically included on routine cervical spine MRI images), if the vessels are not diligently evaluated.

\section{Compliance with ethical standards}

Conflict of interest The authors declare that they have no conflict of interest.

Human and animal rights statement This article does not contain any studies with human participants or animals performed by any of the authors.

Informed consent For this type of study formal consent is waived.

\section{References}

1. Schievink WI, Roiter V (2005) Epidemiology of cervical artery dissection. Front Neurol Neurosci 20:12-15

2. Biousse V, Touboul PJ, D'Anglejan-Chatillon J, Lévy C, Schaison M, Bousser MG (1998) Ophthalmologic manifestations of internal carotid artery dissection. Am J Ophthalmol 126(4):565-577

3. Pandey S, Hakky M, Kwak E, Jara H, Geyer CA, Erbay SH (2013) Application of basic principles of physics to head and neck MR angiography: troubleshooting for artifacts. Radiographics 33(3):E113-E123. doi:10.1148/rg.333125148

4. Kitanaka C, Tanaka J, Kuwahara M, Teraoka A (1994) Magnetic resonance imaging study of intracranial vertebrobasilar artery dissections. Stroke 25(3):571-575 